\title{
Modules of finite homological dimension with respect to a semidualizing module
}

\author{
SeAn Sather-Wagstaff And Siamak Yassemi
}

\begin{abstract}
We prove versions of results of Foxby and Holm about modules of finite (Gorenstein) injective dimension and finite (Gorenstein) projective dimension with respect to a semidualizing module. We also verify special cases of a question of Takahashi and White.
\end{abstract}

Mathematics Subject Classification (2000). 13C05, 13D05, $13 \mathrm{H} 10$.

Keywords. Dualizing modules, Gorenstein homological dimensions, Gorenstein injective dimension, Gorenstein projective dimension, Gorenstein rings, Semidualizing modules.

0. Introduction. Let $R$ be a commutative noetherian ring. It is well-known that, if $R$ is Gorenstein and local, then every module with finite projective dimension has finite injective dimension. Conversely, Foxby $[4,5]$ showed that, if $R$ is local and admits a finitely generated module of finite projective dimension and finite injective dimension, then $R$ is Gorenstein. More recently, Holm [12] proved that, if $M$ is an $R$-module of finite projective dimension and finite Gorenstein injective dimension, then $M$ has finite injective dimension, and so the localization $R_{\mathfrak{p}}$ is Gorenstein for each $\mathfrak{p} \in \operatorname{Spec}(R)$ with $\operatorname{depth}_{R_{\mathfrak{p}}}\left(M_{\mathfrak{p}}\right)<\infty$. See Sect. 1 for terminology and notation.

In this paper, we prove analogues of these results for homological dimensions defined in terms of semidualizing $R$-modules. For instance, the following result is proved in (2.1). Other variants of this result are also given in Sect. 2 . It should be noted that our proof of this result is different from Holm's proof for the special case $C=R$. In particular, this paper also provides a new proof of Holm's result.

Theorem A. Let $C$ be a semidualizing $R$-module, and let $M$ be an $R$-module with $\mathcal{P}_{C}-\operatorname{pd}_{R}(M)<\infty$ and $\operatorname{Gid}_{R}(M)<\infty$. Then $\operatorname{id}_{R}(M)=\operatorname{Gid}_{R}(M)<\infty$

This research was conducted while S.S.-W. visited the IPM in Tehran during July 2008. The research of S.Y. was supported in part by a grant from the IPM (No. 87130211). 
and, for each $\mathfrak{p} \in \operatorname{Spec}(R)$ with $\operatorname{depth}_{R_{\mathfrak{p}}}\left(M_{\mathfrak{p}}\right)$ finite, the $R_{\mathfrak{p}}$-module $C_{\mathfrak{p}}$ is dualizing.

Takahashi and White [19] posed the following question: When $R$ is a local Cohen-Macaulay ring admitting a dualizing module and $C$ is a semidualizing $R$-module, if $M$ is an $R$-module of finite depth such that $\mathcal{P}_{C}-\operatorname{pd}_{R}(M)$ and $\mathcal{I}_{C^{-}} \operatorname{id}_{R}(M)$ are finite, must $R$ be Gorenstein? An affirmative answer to this question would yield another generalization of Foxby's theorem. Our techniques allow us to answer the question in the affirmative in three special cases. The first one is contained in the next result which we prove in (2.13); the others are in Theorem 2.14 and Corollary 2.15.

Theorem B. Let $C$ be a semidualizing $R$-module, and let $M$ be an $R$-module with $\mathcal{P}_{C}-\operatorname{pd}_{R}(M)=0$ and $\mathcal{I}_{C}-\operatorname{id}_{R}(M)<\infty$. Then $R_{\mathfrak{p}}$ is Gorenstein for each $\mathfrak{p} \in \operatorname{Supp}_{R}(M)$.

1. Semidualizing modules and related homological dimensions. Throughout this paper $R$ is a commutative noetherian ring.

This section contains definitions and background information for use in the proofs of our main results in Sect. 2 .

Definition 1.1. Let $\mathcal{X}$ be a class of $R$-modules and $M$ an $R$-module. An $\mathcal{X}$-resolution of $M$ is a complex of $R$-modules in $\mathcal{X}$ of the form

$$
X=\cdots \stackrel{\partial_{n+1}^{X}}{\longrightarrow} X_{n} \stackrel{\partial_{n}^{X}}{\longrightarrow} X_{n-1} \stackrel{\partial_{n-1}^{X}}{\longrightarrow} \cdots \stackrel{\partial_{1}^{X}}{\longrightarrow} X_{0} \rightarrow 0
$$

such that $\mathrm{H}_{0}(X) \cong M$ and $\mathrm{H}_{n}(X)=0$ for $n \geqslant 1$. The $\mathcal{X}$-projective dimension of $M$ is the quantity.

$$
\mathcal{X}-\operatorname{pd}_{R}(M)=\inf \left\{\sup \left\{n \geqslant 0 \mid X_{n} \neq 0\right\} \mid X \text { is an } \mathcal{X} \text {-resolution of } M\right\} .
$$

In particular, one has $\mathcal{X}-\operatorname{pd}_{R}(0)=-\infty$. The modules of $\mathcal{X}$-projective dimension 0 are the nonzero modules of $\mathcal{X}$.

Dually, an $\mathcal{X}$-coresolution of $M$ is a complex of $R$-modules in $\mathcal{X}$ of the form

$$
X=0 \rightarrow X_{0} \stackrel{\partial_{0}^{X}}{\longrightarrow} X_{-1} \stackrel{\partial_{-1}^{X}}{\longrightarrow} \cdots \stackrel{\partial_{n+1}^{X}}{\longrightarrow} X_{n} \stackrel{\partial_{n}^{X}}{\longrightarrow} X_{n-1} \stackrel{\partial_{n-1}^{X}}{\longrightarrow} \cdots
$$

such that $\mathrm{H}_{0}(X) \cong M$ and $\mathrm{H}_{n}(X)=0$ for $n \leqslant-1$. The $\mathcal{X}$-injective dimension of $M$ is the quantity

$$
\mathcal{X} \text {-id } \operatorname{id}_{R}(M)=\inf \left\{\sup \left\{-n \geqslant 0 \mid X_{n} \neq 0\right\} \mid X \text { is an } \mathcal{X} \text {-coresolution of } M\right\} .
$$

In particular, one has $\mathcal{X}-\operatorname{id}_{R}(0)=-\infty$. The modules of $\mathcal{X}$-injective dimension 0 are the nonzero modules of $\mathcal{X}$.

When $\mathcal{X}$ is the class of projective $R$-modules, we write $\operatorname{pd}_{R}(M)$ for the associated homological dimension and call it the projective dimension of $M$. Similarly, the flat and injective dimensions of $M$ are denoted $\operatorname{fd}_{R}(M)$ and $\operatorname{id}_{R}(M)$.

The homological dimensions of interest in this paper are built from semidualizing modules and their associated projective and injective classes, defined next. Semidualizing modules occur in the literature with several different names, e.g., in the work of Foxby [3], Golod [9], Mantese and Reiten [17], 
Vasconcelos [20] and Wakamatsu [21]. The prototypical semidualizing modules are the dualizing (or canonical) modules of Grothendieck and Hartshorne [10].

Definition 1.2. A finitely generated $R$-module $C$ is semidualzing if the natural homothety morphism $R \rightarrow \operatorname{Hom}_{R}(C, C)$ is an isomorphism and $\operatorname{Ext}_{R}^{\geqslant 1}(C, C)=0$. An $R$-module $D$ is dualizing if it is semidualizing and has finite injective dimension.

Let $C$ be a semidualizing $R$-module. We set

$\mathcal{P}_{C}(R)=$ the subcategory of modules $P \otimes_{R} C$ where $P$ is $R$-projective

$\mathcal{F}_{C}(R)=$ the subcategory of modules $F \otimes_{R} C$ where $F$ is $R$-flat

$\mathcal{I}_{C}(R)=$ the subcategory of modules $\operatorname{Hom}_{R}(C, I)$ where $I$ is $R$-injective.

Modules in $\mathcal{P}_{C}(R)$ are called $C$-projective, and those in $\mathcal{I}_{C}(R)$ are $C$-injective.

Fact 1.3. Let $C$ be a semidualizing $R$-module. It is straightforward to show that, if $P \in \mathcal{P}_{C}(R)$ and $I \in \mathcal{I}_{C}(R)$, then $P_{\mathfrak{p}} \in \mathcal{P}_{C_{\mathfrak{p}}}\left(R_{\mathfrak{p}}\right)$ and $I_{\mathfrak{p}} \in \mathcal{I}_{C_{\mathfrak{p}}}\left(R_{\mathfrak{p}}\right)$ for each $\mathfrak{p} \in \operatorname{Spec}(R)$. It follows that we have $\mathcal{P}_{C_{\mathfrak{p}}}-\operatorname{pd}_{R_{\mathfrak{p}}}\left(M_{\mathfrak{p}}\right) \leqslant \mathcal{P}_{C^{-}} \operatorname{pd}_{R}(M)$ and $\mathcal{I}_{C_{\mathfrak{p}}}-\operatorname{id}_{R_{\mathfrak{p}}}\left(M_{\mathfrak{p}}\right) \leqslant \mathcal{I}_{C^{-}} \mathrm{id}_{R}(M)$ for each $R$-module $M$.

Fact 1.4. A result of Gruson and Raynaud [18, Seconde Partie, Théorème (3.2.6)], and Jensen [16, Proposition 6] says that, if $F$ is a flat $R$-module, then $\operatorname{pd}_{R}(F) \leqslant \operatorname{dim}(R)$. It follows that, if $\operatorname{dim}(R)<\infty$, then $\mathcal{P}_{C}-\operatorname{pd}_{R}(M)<\infty$.

The next classes are central to our proofs and were introduced by Foxby [6].

Definition 1.5. Let $C$ be a semidualizing $R$-module. The Auslander class of $C$ is the class $\mathcal{A}_{C}(R)$ of $R$-modules $M$ such that

(1) $\operatorname{Tor}_{\geqslant 1}^{R}(C, M)=0=\operatorname{Ext}_{R}^{\geqslant 1}\left(C, C \otimes_{R} M\right)$, and

(2) the natural map $M \rightarrow \operatorname{Hom}_{R}\left(C, C \otimes_{R} M\right)$ is an isomorphism.

The Bass class of $C$ is the class $\mathcal{B}_{C}(R)$ of $R$-modules $M$ such that

(1) $\operatorname{Ext}_{R}^{\geqslant 1}(C, M)=0=\operatorname{Tor}_{\geqslant 1}^{R}\left(C, \operatorname{Hom}_{R}(C, M)\right)$, and

(2) the natural evaluation map $C \otimes_{R} \operatorname{Hom}_{R}(C, M) \rightarrow M$ is an isomorphism.

Fact 1.6. Let $C$ be a semidualizing $R$-module. The categories $\mathcal{A}_{C}(R)$ and $\mathcal{B}_{C}(R)$ are closed under extensions, kernels of epimorphisms and cokernels of monomorphisms; see [14, Corollary 6.3]. The category $\mathcal{A}_{C}(R)$ contains all modules of finite flat dimension and those of finite $\mathcal{I}_{C}$-injective dimension, and the category $\mathcal{B}_{C}(R)$ contains all modules of finite injective dimension and those of finite $\mathcal{P}_{C}$-projective dimension by [14, Corollaries 6.1 and 6.2].

The next definitions are due to Holm and Jørgensen [13] in this generality.

Definition 1.7. Let $C$ be a semidualizing $R$-module. A complete $\mathcal{I}_{C} \mathcal{I}$-resolution is a complex $Y$ of $R$-modules satisfying the following:

(1) $Y$ is exact and $\operatorname{Hom}_{R}(I, Y)$ is exact for each $I \in \mathcal{I}_{C}(R)$, and

(2) $Y_{i} \in \mathcal{I}_{C}(R)$ for all $i \geqslant 0$ and $Y_{i} \in \mathcal{I}(R)$ for all $i<0$. 
An $R$-module $H$ is $G_{C}$-injective if there exists a complete $\mathcal{I}_{C} \mathcal{I}$-resolution $Y$ such that $H \cong \operatorname{Im}\left(\partial_{0}^{Y}\right)$, in which case $Y$ is a complete $\mathcal{I}_{C} \mathcal{I}$-resolution of $H$. We set

$$
\mathcal{G I}_{C}(R)=\text { the class of } \mathrm{G}_{C} \text {-injective } R \text {-modules. }
$$

In the special case $C=R$, we set $\operatorname{Gid}_{R}(M)=\mathcal{G} \mathcal{I}_{R^{-}} \mathrm{id}_{R}(M)$, and we write "complete injective resolution" instead of "complete $\mathcal{I}_{R} \mathcal{I}$-resolution".

A complete $\mathcal{P} \mathcal{P}_{C}$-resolution is a complex $X$ of $R$-modules such that:

(1) $X$ is exact and $\operatorname{Hom}_{R}(X, P)$ is exact for each $P \in \mathcal{P}_{C}(R)$, and

(2) $X_{i} \in \mathcal{P}(R)$ for all $i \geqslant 0$ and $X_{i} \in \mathcal{P}_{C}(R)$ for all $i<0$.

An $R$-module $M$ is $G_{C}$-projective if there exists a complete $\mathcal{P} \mathcal{P}_{C}$-resolution $X$ such that $M \cong \operatorname{Im}\left(\partial_{0}^{X}\right)$, in which case $X$ is a complete $\mathcal{P} \mathcal{P}_{C}$-resolution of $M$. Set

$$
\mathcal{G} \mathcal{P}_{C}(R)=\text { the class of } \mathrm{G}_{C} \text {-projective } R \text {-modules. }
$$

In the case $C=R$, we set $\operatorname{Gpd}_{R}(M)=\mathcal{G} \mathcal{P}_{R^{-}} \operatorname{pd}_{R}(M)$.

The next two lemmas are proved as in $[2,(2.17),(2.18)]$ using tools from $[22]$.

Lemma 1.8. Let $C$ be a semidualizing $R$-module and let $M$ be an $R$-module with $\mathcal{G P} \mathcal{P}_{C}-\operatorname{pd}_{R}(M)<\infty$. There is an exact sequence of $R$-modules

$$
0 \rightarrow M \rightarrow P \rightarrow M^{\prime} \rightarrow 0
$$

such that $M^{\prime} \in \mathcal{G} \mathcal{P}_{C}(R)$ and $\mathcal{P}_{C}-\operatorname{pd}_{R}(P)=\mathcal{G} \mathcal{P}_{C}-\operatorname{pd}_{R}(M)$.

Lemma 1.9. Let $C$ be a semidualizing $R$-module and let $M$ be an $R$-module with $\mathcal{G I}_{C}-\operatorname{id}_{R}(M)<\infty$. There is an exact sequence of $R$-modules

$$
0 \rightarrow M^{\prime} \rightarrow E \rightarrow M \rightarrow 0
$$

such that $\mathcal{I}_{C}-\operatorname{id}_{R}(E)=\mathcal{G I}_{C}-\operatorname{id}_{R}(M)$ and $M^{\prime} \in \mathcal{G I}_{C}(R)$.

Definition 1.10. Assume that $R$ is local with residue field $k$. The depth of a (not necessarily finitely generated) $R$-module $M$ is

$$
\operatorname{depth}_{R}(M)=\inf \left\{n \geqslant 0 \mid \operatorname{Ext}_{R}^{n}(k, M) \neq 0\right\} .
$$

\section{Main results.}

2.1. Proof of Theorem A. As $\operatorname{Gid}_{R}(M)$ is finite, Lemma 1.9 yields an exact sequence of $R$-modules

$$
0 \rightarrow M^{\prime} \rightarrow E \rightarrow M \rightarrow 0
$$

such that $\operatorname{id}_{R}(E)<\infty$ and $M^{\prime}$ is G-injective. The finiteness of $\mathcal{P}_{C}-\operatorname{pd}_{R}(M)$ and $\operatorname{id}_{R}(E)$ implies that $M, E \in \mathcal{B}_{C}(R)$, and so $M^{\prime} \in \mathcal{B}_{C}(R)$; see Fact 1.6.

We claim that $\operatorname{Ext}_{R}^{\geqslant 1}\left(M, M^{\prime}\right)=0$. To see this, let $Y$ be a complete injective resolution of $M^{\prime}$ and set $M^{(i)}=\operatorname{Im}\left(\partial_{i}^{Y}\right)$ for each $i \in \mathbb{Z}$. Since $M^{\prime}, Y_{i} \in \mathcal{B}_{C}(R)$ for each $i \in \mathbb{Z}$, we have $M^{(i)} \in \mathcal{B}_{C}(R)$ for each $i$, and so $\operatorname{Ext}_{R}^{\geqslant 1}\left(C, M^{(i)}\right)=0$. Hence

$$
\operatorname{Ext}_{R}^{\geqslant 1}\left(P \otimes_{R} C, M^{(i)}\right) \cong \operatorname{Hom}_{R}\left(P, \operatorname{Ext}_{R}^{\geqslant 1}\left(C, M^{(i)}\right)\right)=0
$$


for each projective $R$-module $P$ and each $i$. Using a bounded $\mathcal{P}_{C}$-resolution of $M$, a dimension-shifting argument shows that $\operatorname{Ext}_{R}^{\geqslant d+1}\left(M, M^{(i)}\right)=0$ for each $i$ where $d=\mathcal{P}_{C^{-}} \operatorname{pd}_{R}(M)$. Another dimension-shifting argument using the complete injective resolution of $M^{\prime}$ yields the following.

$$
\operatorname{Ext}_{R}^{\geqslant 1}\left(M, M^{\prime}\right) \cong \operatorname{Ext}_{R}^{\geqslant 1}\left(M, M^{(0)}\right) \cong \operatorname{Ext}_{R}^{\geqslant d+1}\left(M, M^{(d)}\right)=0
$$

as claimed.

The previous paragraph shows that the sequence $(*)$ splits. Hence, we have

$$
\sup \left\{\operatorname{id}_{R}(M), \operatorname{id}_{R}\left(M^{\prime}\right)\right\}=\operatorname{id}_{R}(E)<\infty
$$

and so $\operatorname{id}_{R}(M)<\infty$. The equality $\operatorname{id}_{R}(M)=\operatorname{Gid}_{R}(M)$ now follows from the result dual to $[11,(2.27)]$.

Now, let $\mathfrak{p} \in \operatorname{Spec}(R)$ with $\operatorname{depth}_{R_{\mathfrak{p}}}\left(M_{\mathfrak{p}}\right)$ finite. Using Fact 1.3 we conclude that $\mathcal{P}_{C_{\mathfrak{p}}}-\mathrm{pd}_{R_{\mathfrak{p}}}\left(M_{\mathfrak{p}}\right)$ and $\operatorname{id}_{R_{\mathfrak{p}}}\left(M_{\mathfrak{p}}\right)$ are finite. The finiteness of $\mathcal{P}_{C_{\mathfrak{p}}}-\operatorname{pd}_{R_{\mathfrak{p}}}\left(M_{\mathfrak{p}}\right)$ implies $M_{\mathfrak{p}} \in \mathcal{B}_{C_{\mathfrak{p}}}\left(R_{\mathfrak{p}}\right)$ and thus $\operatorname{Ext}_{R_{\mathfrak{p}}}^{\geqslant 1}\left(C_{\mathfrak{p}}, M_{\mathfrak{p}}\right)=0$. (Hence, in the derived category $\mathcal{D}\left(R_{\mathfrak{p}}\right)$, there is an isomorphism $\mathbf{R H o m}_{R_{\mathfrak{p}}}\left(C_{\mathfrak{p}}, M_{\mathfrak{p}}\right) \simeq \operatorname{Hom}_{R_{\mathfrak{p}}}\left(C_{\mathfrak{p}}, M_{\mathfrak{p}}\right)$ ). Using $[19,(2.11 . c)]$, the finiteness of $\mathcal{P}_{C_{\mathfrak{p}}}-\mathrm{pd}_{R_{\mathfrak{p}}}\left(M_{\mathfrak{p}}\right)$ also implies

$$
\operatorname{fd}_{R_{\mathfrak{p}}}\left(\operatorname{Hom}_{R_{\mathfrak{p}}}\left(C_{\mathfrak{p}}, M_{\mathfrak{p}}\right)\right) \leqslant \operatorname{pd}_{R_{\mathfrak{p}}}\left(\operatorname{Hom}_{R_{\mathfrak{p}}}\left(C_{\mathfrak{p}}, M_{\mathfrak{p}}\right)\right)=\mathcal{P}_{C_{\mathfrak{p}}}-\operatorname{pd}_{R_{\mathfrak{p}}}\left(M_{\mathfrak{p}}\right)<\infty .
$$

The $R_{\mathfrak{p}}$-module $M_{\mathfrak{p}}$ has finite injective dimension and finite depth, so the finiteness of $\operatorname{fd}_{R_{\mathfrak{p}}}\left(\operatorname{Hom}_{R_{\mathfrak{p}}}\left(C_{\mathfrak{p}}, M_{\mathfrak{p}}\right)\right)$ implies that $C_{\mathfrak{p}}$ is dualizing for $R_{\mathfrak{p}}$; see $[1,(8.2)]$.

Corollary 2.2. Assume that $R$ is local, and let $C$ be a semidualizing $R$-module. The following conditions are equivalent:

(i) $C$ is a dualizing $R$-module;

(ii) there exists a finitely generated $R$-module $M \neq 0$ such that $\mathcal{P}_{C}-\operatorname{pd}_{R}(M)$ $<\infty$ and $\operatorname{id}_{R}(M)<\infty$

(iii) there exists an $R$-module $M \neq 0$ of finite depth such that $\mathcal{P}_{C}-\operatorname{pd}_{R}(M)<$ $\infty$ and $\operatorname{Gid}_{R}(M)<\infty$.

Proof. The implication (ii) $\Longrightarrow$ (iii) is straightforward, and (iii) $\Longrightarrow$ (i) follows from Theorem A. For (i) $\Longrightarrow\left(\right.$ ii), note that $\mathcal{P}_{C^{-}} \operatorname{pd}_{R}(C)<\infty$ and $\operatorname{id}_{R}(C)<\infty$ since $C$ is dualizing for $R$.

The following versions of Theorem A and Corollary 2.2 are proved similarly, using Lemmas 1.8 and 1.9.

Theorem 2.3. Let $C$ be a semidualizing $R$-module, and let $M$ be an $R$-module with $\operatorname{pd}_{R}(M)<\infty$ and $\mathcal{G} \mathcal{I}_{C}-\operatorname{id}_{R}(M)<\infty$. Then $\mathcal{I}_{C}-\mathrm{id}_{R}(M)=\mathcal{G I}_{C}-\mathrm{id}_{R}(M)<$ $\infty$. Furthermore, for each $\mathfrak{p} \in \operatorname{Spec}(R)$ such that $\operatorname{depth}_{R_{\mathfrak{p}}}\left(M_{\mathfrak{p}}\right)$ is finite, the localization $C_{\mathfrak{p}}$ is a dualizing $R_{\mathfrak{p}}$-module.

Corollary 2.4. Assume that $R$ is local, and let $C$ be a semidualizing $R$-module. The following conditions are equivalent:

(i) $C$ is a dualizing $R$-module;

(ii) there exists a finitely generated $R$-module $M \neq 0$ such that $\operatorname{pd}_{R}(M)<\infty$ and $\mathcal{I}_{C}-\mathrm{id}_{R}(M)<\infty$; 
(iii) there exists an $R$-module $M \neq 0$ of finite depth such that $\operatorname{pd}_{R}(M)<\infty$ and $\mathcal{G I}_{C}-\mathrm{id}_{R}(M)<\infty$.

Theorem 2.5. Let $C$ be a semidualizing $R$-module, and let $M$ be an $R$-module with $\mathcal{I}_{C}-\mathrm{id}_{R}(M)<\infty$ and $\operatorname{Gpd}_{R}(M)<\infty$. Then $\operatorname{pd}_{R}(M)=\operatorname{Gpd}_{R}(M)<$ $\infty$. Furthermore, for each $\mathfrak{p} \in \operatorname{Spec}(R)$ such that $\operatorname{depth}_{R_{\mathfrak{p}}}\left(M_{\mathfrak{p}}\right)$ is finite, the localization $C_{\mathfrak{p}}$ is a dualizing $R_{\mathfrak{p}}$-module.

Corollary 2.6. Assume that $R$ is local, and let $C$ be a semidualizing $R$-module. The following conditions are equivalent:

(i) $C$ is a dualizing $R$-module;

(ii) there exists a finitely generated $R$-module $M \neq 0$ such that $\mathcal{I}_{C}$ - $\operatorname{id}_{R}(M)<$ $\infty$ and $\operatorname{pd}_{R}(M)<\infty$;

(iii) there exists an $R$-module $M \neq 0$ of finite depth such that $\mathcal{I}_{C}-\operatorname{id}_{R}(M)<\infty$ and $\operatorname{Gpd}_{R}(M)<\infty$.

Remark 2.7. As is noted in [12], when $R$ has finite Krull dimension, we can change $\operatorname{Gpd}_{R}(M)$ and $\operatorname{pd}_{R}(M)$ to $\operatorname{Gfd}_{R}(M)$ and $\mathrm{fd}_{R}(M)$, respectively, in the previous two results. Similarly, in the next two results, if $\operatorname{dim}(R)<\infty$, then $\mathcal{G} \mathcal{P}_{C^{-}} \operatorname{pd}_{R}(M)$ and $\mathcal{P}_{C^{-}} \operatorname{pd}_{R}(M)$ can be changed to $\mathcal{G} \mathcal{F}_{C^{-}} \operatorname{pd}_{R}(M)$ and $\mathcal{F}_{C^{-}} \operatorname{pd}_{R}(M)$.

Theorem 2.8. Let $C$ be a semidualizing $R$-module, and let $M$ be an $R$-module with $\operatorname{id}_{R}(M)<\infty$ and $\mathcal{G P} \mathcal{P}_{C}-\operatorname{pd}_{R}(M)<\infty$. Then $\mathcal{P}_{C}-\operatorname{pd}_{R}(M)=$ $\mathcal{G} \mathcal{P}_{C}-\operatorname{pd}_{R}(M)<\infty$. Furthermore, for each $\mathfrak{p} \in \operatorname{Spec}(R)$ such that $\operatorname{depth}_{R_{\mathfrak{p}}}\left(M_{\mathfrak{p}}\right)$ is finite, the localization $C_{\mathfrak{p}}$ is a dualizing $R_{\mathfrak{p}}$-module.

Corollary 2.9. Assume that $R$ is local, and let $C$ be a semidualizing $R$-module. The following conditions are equivalent:

(i) $C$ is a dualizing $R$-module;

(ii) there exists a finitely generated $R$-module $M \neq 0$ such that $\operatorname{id}_{R}(M)<\infty$ and $\mathcal{P}_{C}-\operatorname{pd}_{R}(M)<\infty$;

(iii) there exists an $R$-module $M \neq 0$ of finite depth such that $\operatorname{id}_{R}(M)<\infty$ and $\mathcal{G P} C-\operatorname{pd}_{R}(M)<\infty$.

Remark 2.10. Holm proves his results in a more general setting than ours, namely, over associative rings. While the Gorenstein projective dimension and Gorenstein injective dimension have been well-studied in this setting, the same cannot be said for $\mathrm{G}_{C}$-projective dimension and $\mathrm{G}_{C}$-injective dimension. Some of the foundation has been laid by Holm and White [14]. To prove our results in this setting, though, would require a development of these ideas that is outside the scope of this paper.

The next lemma is useful for the two subsequent proofs.

Lemma 2.11. Let $C$ be a semidualizing $R$-module. If $\mathcal{I}_{C}-\mathrm{id}_{R}(C)<\infty$, then $C \cong R$ and $R$ is Gorenstein. 
Proof. Assume that $\mathcal{I}_{C^{-}} \mathrm{id}_{R}(C)<\infty$. Fact 1.6 implies that $C \in \mathcal{A}_{C}(R)$. By definition, this includes the condition $\operatorname{Tor}_{\geqslant 1}^{R}(C, C)=0$, and so $[8,(3.8)]$ implies that $C \otimes_{R} C$ is a semidualizing $R$-module. From [7, (3.2)] we conclude that $C \cong R$. It follows that $\operatorname{id}_{R}(R)=\mathcal{I}_{C}-\operatorname{id}_{R}(C)<\infty$ and so $R$ is Gorenstein as desired.

Remark 2.12. In unpublished work, Takahashi and White have proved the following result that is weaker than Theorem B: If $R$ is Cohen-Macaulay with a dualizing module $D$ and $C$ is a semidualizing module with $\mathcal{I}_{C}-\operatorname{id}_{R}(C)<\infty$, then $C \cong D$.

2.13. Proof of Theorem B. Let $\mathfrak{p} \in \operatorname{Supp}_{R}(M)$, and replace $R$ with $R_{\mathfrak{p}}$ to assume that $R$ is local. In particular, every projective $R$-module is free, and so $M \cong C \oplus M^{\prime}$ for some $M^{\prime} \in \mathcal{P}_{C}(R)$. In the next sequence, the final equality is from $[19,(2.11 . b)]$

$$
\begin{aligned}
\sup \left\{\operatorname{id}_{R}\left(C \otimes_{R} C\right), \operatorname{id}_{R}\left(C \otimes_{R} M^{\prime}\right)\right\} & =\operatorname{id}_{R}\left(\left(C \otimes_{R} C\right) \oplus\left(C \otimes_{R} M^{\prime}\right)\right) \\
& =\operatorname{id}_{R}\left(C \otimes_{R}\left(C \oplus M^{\prime}\right)\right) \\
& =\operatorname{id}_{R}\left(C \otimes_{R} M\right) \\
& =\mathcal{I}_{C^{-}} \operatorname{id}_{R}(M)
\end{aligned}
$$

and so $\mathcal{I}_{C^{-}} \operatorname{id}_{R}(C) \leqslant \mathcal{I}_{C^{-}} \operatorname{id}_{R}(M)<\infty$. Lemma 2.11 implies that $R$ is Gorenstein, as desired.

The next result contains another partial answer to the question of Takahashi and White. We include the proof as it is different from the proof of Theorem B.

Theorem 2.14. If $C$ is a semidualizing $R$-module and $M$ is an $R$-module such that $\mathcal{P}_{C}-\operatorname{pd}_{R}(M)<\infty$ and $\mathcal{I}_{C}-\operatorname{id}_{R}(M)=0$, then $R_{\mathfrak{p}}$ is Gorenstein for all $\mathfrak{p} \in \operatorname{Spec}(R)$ such that $\operatorname{depth}_{R_{\mathfrak{p}}}\left(M_{\mathfrak{p}}\right)$ is finite.

Proof. As $M \in \mathcal{I}_{C}(R)$, we have $M \cong \operatorname{Hom}_{R}(C, E)$ for some injective $R$-module $E$.

We first show that the assumption that $\operatorname{depth}_{R_{\mathfrak{p}}}\left(M_{\mathfrak{p}}\right)$ is finite implies that $\mathfrak{p} \in \operatorname{Ass}_{R}(M)$. The fact that $C$ is finitely generated and $E$ is injective yields the next isomorphisms

$$
\begin{aligned}
\operatorname{Ext}_{R_{\mathfrak{p}}}^{i}\left(R_{\mathfrak{p}} / \mathfrak{p} R_{\mathfrak{p}}, M_{\mathfrak{p}}\right) & \cong \operatorname{Ext}_{R_{\mathfrak{p}}}^{i}\left(R_{\mathfrak{p}} / \mathfrak{p} R_{\mathfrak{p}}, \operatorname{Hom}_{R}(C, E)_{\mathfrak{p}}\right) \\
& \cong \operatorname{Ext}_{R_{\mathfrak{p}}}^{i}\left(R_{\mathfrak{p}} / \mathfrak{p} R_{\mathfrak{p}}, \operatorname{Hom}_{R_{\mathfrak{p}}}\left(C_{\mathfrak{p}}, E_{\mathfrak{p}}\right)\right) \\
& \cong \operatorname{Hom}_{R_{\mathfrak{p}}}\left(\operatorname{Tor}_{i}^{R_{\mathfrak{p}}}\left(R_{\mathfrak{p}} / \mathfrak{p} R_{\mathfrak{p}}, C_{\mathfrak{p}}\right), E_{\mathfrak{p}}\right)
\end{aligned}
$$

Each module $\operatorname{Tor}_{i}^{R_{\mathfrak{p}}}\left(R_{\mathfrak{p}} / \mathfrak{p} R_{\mathfrak{p}}, C_{\mathfrak{p}}\right)$ is a finite-dimensional vector space over $R_{\mathfrak{p}} / \mathfrak{p} R_{\mathfrak{p}}$. Furthermore, we have $\operatorname{Tor}_{0}^{R_{\mathfrak{p}}}\left(R_{\mathfrak{p}} / \mathfrak{p} R_{\mathfrak{p}}, C_{\mathfrak{p}}\right) \cong R_{\mathfrak{p}} / \mathfrak{p} R_{\mathfrak{p}} \otimes_{R_{\mathfrak{p}}} C_{\mathfrak{p}} \neq 0$ since $C_{\mathfrak{p}}$ is nonzero and finitely generated over $R_{\mathfrak{p}}$. Since $\operatorname{Ext}_{R_{\mathfrak{p}}}^{i}\left(R_{\mathfrak{p}} / \mathfrak{p} R_{\mathfrak{p}}, M_{\mathfrak{p}}\right) \neq 0$ 
for some $i$, by hypothesis, it therefore follows that $\operatorname{Ext}_{R_{\mathfrak{p}}}^{0}\left(R_{\mathfrak{p}} / \mathfrak{p} R_{\mathfrak{p}}, M_{\mathfrak{p}}\right) \neq 0$, and so $\mathfrak{p} R_{\mathfrak{p}} \in \operatorname{Ass}_{R_{\mathfrak{p}}}\left(M_{\mathfrak{p}}\right)$, that is, $\mathfrak{p} \in \operatorname{Ass}_{R}(M)$ as claimed.

Write $E \cong \oplus_{\mathfrak{q}} E_{R}(R / \mathfrak{q})^{\left(\mu_{\mathfrak{q}}\right)}$, where the direct sum is taken over all $\mathfrak{q} \in$ $\operatorname{Spec}(R)$. It follows that there are equalities

$$
\begin{aligned}
& \operatorname{Ass}_{R}(M)=\operatorname{Supp}_{R}(C) \cap \operatorname{Ass}_{R}(E)=\operatorname{Spec}(R) \cap \operatorname{Ass}_{R}(E) \\
& \quad=\left\{\mathfrak{q} \in \operatorname{Spec}(R) \mid \mu_{\mathfrak{q}} \neq 0\right\} .
\end{aligned}
$$

Since $\mathfrak{p} \in \operatorname{Ass}_{R}(M)$, this implies $E \cong E_{R}(R / \mathfrak{p}) \oplus E^{\prime}$ for some injective $R$-module $E^{\prime}$. It follows that $M \cong \operatorname{Hom}_{R}\left(C, E_{R}(R / \mathfrak{p})\right) \oplus \operatorname{Hom}_{R}\left(C, E^{\prime}\right)$. As in the proof of Theorem B, using $[19,(2.11 . c)]$ we see that $\mathcal{P}_{C^{-}} \operatorname{pd}_{R}\left(\operatorname{Hom}_{R}\left(C, E_{R}(R / \mathfrak{p})\right)\right)<$ $\infty$. If follows that we may replace $R$ with $R_{\mathfrak{p}}$ and $M$ with $\operatorname{Hom}_{R}\left(C, E_{R}(R / \mathfrak{p})\right)_{\mathfrak{p}}$ to assume that $R$ is local with maximal ideal $\mathfrak{m}$ and $M \cong \operatorname{Hom}_{R}(C, E)$ where $E=E_{R}(R / \mathfrak{m})$.

Let $\widehat{R}$ denote the completion of $R$. It is straightforward to show that the condition $\mathcal{P}_{C^{-}} \operatorname{pd}_{R}(M)<\infty$ implies $\mathcal{P}_{\widehat{C}^{-}} \operatorname{pd}_{\widehat{R}}\left(M \otimes_{R} \widehat{R}\right)<\infty$. Also, we have isomorphisms

$$
M \otimes_{R} \widehat{R} \cong \operatorname{Hom}_{R}\left(C, E_{R}(R / \mathfrak{m})\right) \otimes_{R} \widehat{R} \cong \operatorname{Hom}_{\widehat{R}}\left(\widehat{C}, E_{\widehat{R}}(\widehat{R} / \mathfrak{m} \widehat{R})\right)
$$

and so $M \otimes_{R} \widehat{R} \in \mathcal{I}_{\widehat{C}}(\widehat{R})$. It follows that we may replace $R$ with $\widehat{R}$ and $M$ with $M \otimes_{R} \widehat{R}$ to assume that $R$ is complete.

To complete the proof, we show that $\mathcal{I}_{C}$-id ${ }_{R}(C)<\infty$; the desired conclusion then follows from Lemma 2.11. The module $M$ admits a bounded augmented $\mathcal{P}_{C}$-resolution

$$
0 \rightarrow C \otimes_{R} P_{n} \rightarrow \cdots \rightarrow C \otimes_{R} P_{0} \rightarrow M \rightarrow 0 .
$$

Applying the functor $\operatorname{Hom}_{R}(-, E)$ yields an exact sequence

$$
0 \rightarrow \underbrace{\operatorname{Hom}_{R}(M, E)}_{\cong C} \rightarrow \operatorname{Hom}_{R}\left(C \otimes_{R} P_{0}, E\right) \rightarrow \cdots \rightarrow \operatorname{Hom}_{R}\left(C \otimes_{R} P_{n}, E\right) \rightarrow 0 .
$$

The isomorphism $\operatorname{Hom}_{R}(M, E) \cong C$ follows from Matlis duality because of the assumption $M \cong \operatorname{Hom}_{R}(C, E)$. Since each $P_{i}$ is projective, each module $\operatorname{Hom}_{R}\left(P_{i}, E\right)$ is injective, and so

$$
\operatorname{Hom}_{R}\left(C \otimes_{R} P_{i}, E\right) \cong \operatorname{Hom}_{R}\left(C, \operatorname{Hom}_{R}\left(P_{i}, E\right)\right) \in \mathcal{I}_{C}(R) .
$$

It follows that the displayed exact sequence is an augmented $\mathcal{I}_{C}$-coresolution of $C$, and so $\mathcal{I}_{C}-\operatorname{id}_{R}(C)<\infty$, as desired.

For our final result, recall that, when $R$ is local with maximal ideal $\mathfrak{m}$, the width of an $R$-module $M$ is $\operatorname{width}_{R}(M)=\inf \left\{i \geqslant 0 \mid \operatorname{Tor}_{i}^{R}(k, M) \neq 0\right\}$ where $k=R / \mathfrak{m}$.

Corollary 2.15. If $C$ is a semidualizing $R$-module and $M$ is an $R$-module such that $\mathcal{F}_{C}-\operatorname{pd}_{R}(M)=0$ and $\mathcal{I}_{C}-\operatorname{id}_{R}(M)<\infty$, then $R_{\mathfrak{p}}$ is Gorenstein for all $\mathfrak{p} \in$ $\operatorname{Spec}(R)$ such that width $_{R_{\mathfrak{p}}}\left(M_{\mathfrak{p}}\right)$ is finite.

Proof. As in the proof of Theorem B, replace $R$ and $M$ with $R_{\mathfrak{p}}$ and $M_{\mathfrak{p}}$ to assume that $R$ is local and that $\operatorname{width}_{R}(M)$ is finite. It remains to show that $R$ is Gorenstein. Let $E$ denote the injective hull of the residue field of $R$, and 
set $(-)^{\vee}=\operatorname{Hom}_{R}(-, E)$. By assumption, there is a flat $R$-module $F$ such that $M \cong F \otimes_{R} C$. Hom-tensor adjointness can be used to show that the $R$-module $F^{\vee}$ is injective, and so the sequence of isomorphisms

$$
M^{\vee} \cong \operatorname{Hom}_{R}\left(F \otimes_{R} C, E\right) \cong \operatorname{Hom}_{R}\left(C, \operatorname{Hom}_{R}(F, E)\right)=\operatorname{Hom}_{R}\left(C, F^{\vee}\right)
$$

shows that $M^{\vee} \in \mathcal{I}_{C}(R)$.

We claim that $\mathcal{P}_{C^{-}} \operatorname{pd}_{R}\left(M^{\vee}\right)<\infty$. To see this, consider a bounded augmented $\mathcal{I}_{C}$-coresolution of $M$

$$
0 \rightarrow M \rightarrow \operatorname{Hom}_{R}\left(C, I^{0}\right) \rightarrow \operatorname{Hom}_{R}\left(C, I^{1}\right) \rightarrow \cdots \rightarrow \operatorname{Hom}_{R}\left(C, I^{n}\right) \rightarrow 0 .
$$

The functor $(-)^{\vee}$ yields an exact sequence

$$
0 \rightarrow \operatorname{Hom}_{R}\left(C, I^{n}\right)^{\vee} \rightarrow \cdots \rightarrow \operatorname{Hom}_{R}\left(C, I^{1}\right)^{\vee} \rightarrow \operatorname{Hom}_{R}\left(C, I^{0}\right)^{\vee} \rightarrow M^{\vee} \rightarrow 0 .
$$

For each $j$, the module $\left(I^{j}\right)^{\vee}$ is flat by $[15,(1.5)]$, and Hom-evaluation $[15$, (1.6)] explains the isomorphism in the next display

$\operatorname{Hom}_{R}\left(C, I^{j}\right)^{\vee}=\operatorname{Hom}_{R}\left(\operatorname{Hom}_{R}\left(C, I^{j}\right), E\right) \cong C \otimes_{R} \operatorname{Hom}_{R}\left(I^{j}, E\right)=C \otimes_{R}\left(I^{j}\right)^{\vee}$. It follows that $\operatorname{Hom}_{R}\left(C, I^{j}\right)^{\vee} \in \mathcal{F}_{C}(R)$, and so the sequence $(\dagger)$ implies that $\mathcal{F}_{C^{-}} \operatorname{pd}_{R}\left(M^{\vee}\right)$ is finite. Since $R$ is local, it follows from Fact 1.4 that $\mathcal{P}_{C^{-}} \operatorname{pd}_{R}$ $\left(M^{\vee}\right)$ is also finite, as claimed.

Next, we claim that $\operatorname{depth}_{R}\left(M^{\vee}\right)<\infty$. (Once this is shown, it follows that $R$ is Gorenstein by Theorem 2.14, using the module $M^{\vee}$.) To verify the claim, it suffices to show that $\operatorname{Ext}_{R}^{i}\left(k, M^{\vee}\right) \neq 0$ for some $i$. The assumption width $_{R}(M)<\infty$ implies $\operatorname{Tor}_{i}^{R}(k, M)$ is a nonzero $k$-vector space for some $i$. Write $\operatorname{Tor}_{i}^{R}(k, M) \cong \oplus_{\lambda \in \Lambda} k$ for some index set $\Lambda \neq \emptyset$. The first isomorphism in the next sequence is a version of Hom-tensor adjointness

$$
\operatorname{Ext}_{R}^{i}\left(k, M^{\vee}\right) \cong \operatorname{Tor}_{i}^{R}(k, M)^{\vee} \cong\left(\oplus_{\lambda \in \Lambda} k\right)^{\vee} \cong \prod_{\lambda \in \Lambda} k \vee \cong \prod_{\lambda \in \Lambda} k \neq 0
$$

and the remaining steps are standard.

Acknowledgements. S. S.-W. would like to thank the IPM for its generosity and hospitality during his visit when these results were proved. Both authors are grateful to Diana White for her comments on an earlier version of this manuscript, and to the anonymous referee for useful comments.

\section{References}

[1] L. W. Christensen, Semi-dualizing complexes and their Auslander categories, Trans. Amer. Math. Soc. 353 (2001), 1839-1883.

[2] L. W. Christensen, A. Frankild And H. Holm, On Gorenstein projective, injective and flat dimensions - a functorial description with applications, J. Algebra 302 (2006), 231-279.

[3] H.-B. Foxby, Gorenstein modules and related modules, Math. Scand. 31 (1972), 267-284 (1973).

[4] H.-B. Foxby, Isomorphisms between complexes with applications to the homological theory of modules, Math. Scand. 40 (1977), 5-19. 
[5] H.-B. Foxby, Bounded complexes of flat modules, J. Pure Appl. Algebra 15 (1979), 149-172.

[6] H.-B. Foxby, Gorenstein dimensions over Cohen-Macaulay rings, Proceedings of the international conference on commutative algebra (W. Bruns, ed.), Universität Osnabrück, 1994, pp. 59-63.

[7] A. Frankild and S. Sather-Wagstaff, The set of semidualizing complexes is a nontrivial metric space, J. Algebra 308 (2007), 124-143.

[8] A. J. Frankild, S. Sather-Wagstaff, and A. Taylor, Relations between semidualizing complexes, J. Commut. Algebra, to appear, arXiv:0712.3275v1.

[9] E. S. Golod, G-dimension and generalized perfect ideals, Trudy Mat. Inst. Steklov. 165 (1984), 62-66, Algebraic geometry and its applications.

[10] R. Hartshorne, Local cohomology, A seminar given by A. Grothendieck, Harvard University, Fall 1961, Springer-Verlag, Berlin, 1967.

[11] H. Holm, Gorenstein homological dimensions, J. Pure Appl. Algebra 189 (2004), 167-193.

[12] H. Holm, Rings with finite Gorenstein injective dimension, Proc. Amer. Math. Soc. 132 (2004), 1279-1283 (electronic).

[13] H. Holm and P. Jørgensen, Semi-dualizing modules and related Gorenstein homological dimensions, J. Pure Appl. Algebra 205 (2006), 423-445.

[14] H. Holm And D. White, Foxby equivalence over associative rings, J. Math. Kyoto Univ. 47 (2007), 781-808.

[15] T. Ishikawa, On injective modules and flat modules, J. Math. Soc. Japan 17 (1965), 291-296.

[16] C. U. Jensen, On the vanishing of $\lim ^{(i)}$, J. Algebra 15 (1970), 151-166.

[17] F. Mantese and I. Reiten, Wakamatsu tilting modules, J. Algebra 278 (2004), $532-552$.

[18] M. Raynaud And L. Gruson, Critères de platitude et de projectivité. Techniques de "platification" d'un module, Invent. Math. 13 (1971), 1-89.

[19] R. Takahashi and D. White, Homological aspects of semidualizing modules, Math. Scand., to appear, arXiv:math.AC/0703643v2.

[20] W. V. VAsconcelos, Divisor theory in module categories, North-Holland Publishing Co., Amsterdam, 1974, North-Holland Mathematics Studies, No. 14, Notas de Matemática No. 53. [Notes on Mathematics, No. 53].

[21] T. Wakamatsu, On modules with trivial self-extensions, J. Algebra 114 (1988), 106-114.

[22] D. White, Gorenstein projective dimension with respect to a semidualizing module, J. Commut. Algebra, to appear, arXiv:math.AC/0611711v1.

SeAn SATher-WagstafF

Department of Mathematics, NDSU Dept \# 2750,

PO Box 6050, Fargo, ND 58108-6050, USA

e-mail: Sean. Sather-Wagstaff@ndsu.edu 


\section{SiAmaK YASSEMI}

Department of Mathematics, University of Tehran, P.O. Box 13145-448, Tehran, Iran

\section{SiamaK YASSEMI}

School of Mathematics, Institute for Research in Fundamental Sciences (IPM), P.O. Box 19395-5746, Tehran, Iran

e-mail: yassemi@ipm.ir

Received: 03 December 2008

Revised: 16 March 2009 